\title{
New: Consensus Reports of the European Federation of Conservative Dentistry
}

\author{
Gottfried Schmalz ${ }^{1,2} \cdot$ Adrian Lussi $^{2}$
}

Received: 4 June 2015 / Accepted: 9 June 2015 / Published online: 1 July 2015

(C) Springer-Verlag Berlin Heidelberg 2015

In this issue of Clinical Oral Investigations, you will find the first "Consensus Report" of the European Federation of Conservative Dentistry (EFCD). EFCD unites European national associations for conservative dentistry and prevention, as well as its individual members. Founded in 2001, EFCD organizes biennial scientific meetings, the recent ones 2013 in Paris and 2015 in London. For further information, see the EFCD website: http://www.efcd.eu/.

The EFCD Executive Committee recently decided to issue "Consensus Reports" on clinically relevant topics. These reports are developed in three steps:

1. Literature survey,

2. Formulation of the text of such a report by an expert group, and finally,

3. Approval of this text by the General Assembly of EFCD as a Consensus Report.
The first Consensus Report of the EFCD covers the topic of Erosive Tooth Wear. The EFCD Executive Committee felt that this is a clinically highly relevant topic, and that the information provided in this report may be especially helpful not only in the daily work of the dentist, but also in communicating with patients or institutions like insurances.

As stated in the introduction of the Consensus Report, "The recommendations in this document are not intended to define a standard of care, but rather should be integrated with a practitioner's professional evaluation and judgment, as well as a patient's needs and preferences." Thus, these consensus reports shall help the dentist, but shall not restrict his/her work. With the several consensus reports to come, the EFCD and the Clinical Oral Investigations will help to disseminate standards of care to professionals in dental practices and in universities.

Gottfried Schmalz

Adrian Lussi

Gottfried Schmalz

gottfried.schmalz@klinik.uni-regensburg.de

1 Department of Operative Dentistry and Periodontology, University Medical Centre Regensburg, Franz-Josef-Strauss-Allee 11, 93042 Regensburg, Germany

2 Department of Preventive, Restorative and Pediatric Dentistry, University of Bern, Freiburgstrasse 7, 3010 Bern, Switzerland 